\title{
Selective reduction of extracellular signal-regulated protein kinase (ERK) phosphorylation in squamous cell carcinoma of the larynx
}

\author{
WERNER GARAVELLO ${ }^{1,2}$, GABRIELLA NICOLINI ${ }^{2}$, ALESSIA AGUZZI ${ }^{1}$, DANIELE MAGGIONI ${ }^{2}$, \\ BIAGIO EUGENIO LEONE ${ }^{3}$, PAOLA VIGANOे ${ }^{4}$, RENATO MARIA GAINI ${ }^{1,2}$ and GIOVANNI TREDICI ${ }^{2}$ \\ Departments of ${ }^{1}$ Otorhinolaryngology - Head and Neck Surgery, ${ }^{2}$ Neuroscience and Biomedical Technologies, \\ ${ }^{3}$ Surgical Sciences and Intensive Care, University of Milano-Bicocca, Monza; ${ }^{4}$ Istituto Auxologico Italiano, Milano, Italy
}

Received April 14, 2006; Accepted May 16, 2006

\begin{abstract}
Mitogen-activated protein kinase (MAPK) cascades transmit and amplify signals involved in cell proliferation as well as in cell death. In this study, the potential derangement of MAPK pathways has been evaluated in human squamous cell carcinomas (SCC) of the larynx. The expression and activity of the MAPK p38, ERK $1 / 2^{\mathrm{p} 44 / \mathrm{p}^{42}}$ and JNK/SAPK ${ }^{\mathrm{p} 46 / \mathrm{p} 54}$ have been investigated by immunoblot analysis of tissue homogenates in 27 samples of primary laryngeal cancer and in 27 paired non-neoplastic laryngeal mucosa. On the same tissues, the activation of MAPK JNK/SAPK ${ }^{\mathrm{p} 46 / \mathrm{p} 54}$ was also analyzed by an ELISA assay. The results obtained showed that both total and phosphorylated levels of JNK/SAPK ${ }^{\mathrm{p} 46 / \mathrm{p} 54}$ and p38 were not different between tumor and normal samples. Conversely, while total protein levels for both ERK $1^{\mathrm{p} 44}$ and ERK2 ${ }^{\mathrm{p} 42}$ were not statistically different between tumor and normal samples, the analysis of the level of the activated forms of ERK1/2 showed a statistically significant decreased phosphorylation of both isoforms in the tumor samples compared to the control tissues. The rate of reduction was similar for both isoforms. Immunohistochemical analysis of all the activated MAPK (p38, JNK/SAPK ${ }^{\mathrm{p} 46 / \mathrm{p} 54}$ and ERK1/2 ${ }^{\mathrm{p} 4 / \mathrm{p} 42}$ ) in both laryngeal SCC and normal mucosa demonstrated no difference of cellular localization. Activated ERK1/2p44/p42 and activated p38 demonstrated a nucleo-cytoplasmic distribution whereas activated JNK/SAPK ${ }^{\mathrm{p} 46 / \mathrm{p} 54}$ were localized into the cytoplasmic membrane. The decreased activity of ERK $1 / 2^{\mathrm{p} 44 / 42}$ in laryngeal SCC might reflect alterations in tumor suppressing activity or might derive from the interplay among various transduction pathways.
\end{abstract}

Correspondence to: Dr Werner Garavello, Department of Otorhinolaryngology - Head and Neck surgery, Ospedale San Gerardo, 20052 Monza (MI), Italy

E-mail: werner.garavello@unimib.it

Key words: mitogen-activated protein kinases, laryngeal cancer, squamous cell carcinoma

\section{Introduction}

Squamous cell carcinoma (SCC) of the larynx is the most frequent malignancy occurring in the head and neck region (1). Several epidemiological studies have shown that tobacco and alcohol consumption play a major role in the development of laryngeal SCC (2,3). From a molecular basis, the differences between the normal epithelium of the upper aerodigestive tract and cancer cells arising from that tissue are the result of mutations of the TP53 tumor suppressor gene, overexpression of the epidermal growth factor receptor (EGFR), and of inactivation of the cyclin dependent kinase inhibitor p16 (4-6). These processes control DNA repair, proliferation, immortalization and apoptosis. For head and neck squamous cancers (HNSCC) in general, these alterations occur with sufficient frequency and produce such dramatic phenotypic changes as to be considered the critical transforming events of the disease (7). However, the mechanisms underlying the proliferation of this form of cancer are not fully understood. Controversial findings have, indeed, been reported in regard to the activation and role of the intracellular signal transduction pathways based on mitogen-activated protein kinases (MAPK) $(8,9)$. Moreover, no study has specifically addressed the biologic characteristics of tumors arising from the various anatomic sites of the head and neck area such as those deriving from the larynx.

The MAPK cascade is the most characterized signaling pathway that transmits signals from a variety of stimuli from the cell membrane to the nucleus (10). Activation of the EGFR for instance leads to MAPK activation. In the nucleus, these proteins act on various transcription factors involved in both cell proliferation and apoptosis (10-12). Thus, as supported by their aberrant activation status in several kinds of human cancers, the various MAPK family members play a complex role in determining either cell growth or cell death. The cellular decision is believed to involve a balance among competing MAPK pathways.

The three relatively well-described pathways, which are interconnected, are represented by the extracellular signalregulated kinase (ERK), the JNK/SAPK and p38 pathways. Each cascade is composed of at least three proteins activated in series (13). The ERK1/2 (p44 and p42) pathways are the 
best characterized of the group and are thought to be stimulated predominantly by growth factors. Members of the ERK family were found to be part of a well known oncogenic cascade that includes the proto-oncoproteins Ras and Raf, which act as upstream activators of ERKs (14). Upon activation, ERKs either phosphorylate a number of cytoplasmic targets or migrate into the nucleus where they phosphorylate and activate factors such as c-Fos and Elk-1 (15) regulating the transcription of specific target genes. The c-Jun N-terminal kinases $(\mathrm{JNK} 1 / 2)$ or the stress-activated protein kinase (SAPK) were first identified by their ability to phosphorylate the c-Jun transcription factor following exposure to growth factors or after the expression of transforming oncogenes (16). JNK/SAPK are related to cell stress response and they are known for activating several factors such as ATF2, Bcl2, p53 as well as several oncogenes (17-19). A third group collectively known as p38 are activated by proinflammatory cytokines and chemicals and affect radiation- and druginduced cell death (20).

Because of the propensity of HNSCC in general for EGFR overexpression and the still undefined role of MAPK activation status in their malignant transformation $(8,9)$, we aimed at specifically evaluating laryngeal SCC for potential derangement of one or more of the MAPK pathways leading to uncontrolled proliferation. Thus, in this study we have measured the levels of total MAPKs and the levels of activated (phosphorylated) MAPKs in laryngeal tumors compared with normal tissue obtained from the same patients.

\section{Materials and methods}

Fresh tissue samples were obtained from 27 untreated patients (25 men and 2 women) undergoing surgical resection of a laryngeal neoplasia, histologically confirmed by a previous biopsy, at the Department of Otorhinolaryngology of the San Gerardo Hospital, Monza, Italy, between March 1, 2003, and January 31, 2005. The mean patient age was 67 years (range 52-84 years). None had evidence of neck nodes and/or distant metastases at the time of admission. All the tumor samples were histologically classified as squamous cell carcinomas. Six biopsy specimens were grade 1 (G1), 13 were G2 and 8 were G3. All the patients reported alcohol ( $>11 \mathrm{t} /$ day) and tobacco ( $>20$ cigarettes/day) consumption. For each patient, both a tumoral and a normal control tissue sample was collected. For each case, a tumor sample was resected, avoiding the eventual necrotic center, and a control sample corresponding to a specimen of mucosa was taken from a site adjacent to the resection margins, free from neoplastic infiltration resulting from a subsequent histological examination. Tissue samples were dissected and divided into two specimens, one for the histological and immunohistochemical evaluation, and the other for the immunoblot analysis. Samples for immunoblotting were immediately frozen in liquid nitrogen and stored at $-70^{\circ} \mathrm{C}$. Patients enrolled in this study were informed of the purposes of the study and gave written consent.

Tissues for SDS-PAGE were homogenized on ice in lysis buffer (10\% glycerol, 25 mM Tris- $\mathrm{HCl}$ pH 7.5, 1\% Triton X100, 5 mM EDTA, 1 mM EGTA) containing freshly added protease and phosphatase inhibitors ( $4 \mathrm{mM}$ phenylmethyl- sulfonyl fluoride, $1 \%$ aprotinin, $10 \mathrm{mM}$ sodium orthovanadate, $20 \mathrm{mM}$ sodium pyrophosphate). Protein lysates (60 $\mu \mathrm{g})$ were electrophoresed on $13 \%$ polyacrylamide gel and then transferred onto a nitrocellulose filter. Proteins resolved by SDS-PAGE were transferred to Hybond ECL nitrocellulose membranes (Amersham, Cologno Monzese, Monza, Italy). After brief washing, the membrane was blocked with 5\% not fat milk for $1 \mathrm{~h}$ at room temperature. Immunoblotting was performed with primary polyclonal antibodies antiERK1/2 ${ }^{\mathrm{p} 44 / \mathrm{p} 42}$, anti-phospho-ERK1/2 ${ }^{\mathrm{p} 4 / \mathrm{p} 42}$, anti-p38, anti-

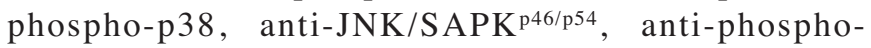
JNK/SAPK ${ }^{\text {p46/p54 }}$ (New England Biolabs Inc., Beverly, MA) and anti-actin (Santa Cruz Biotecnology, CA) followed by a secondary antibody (horseradish peroxidase-conjugated anti-IgG). The blots were visualized using the ECL chemiluminescence system and quantified by densitometric analysis of autoluminographs. Actin immunoblots were performed as loading controls. Results were expressed as relative expression (RE) considering the ratio between the protein band optical density under study to corresponding actin band optical density. The paired Student's t-test was used to compare values of proteins in the paired control and tumor specimens. For the various effectors considered, the levels of expression in each normal tissue were set to 1 and the relative change in expression (fold increase or decrease) in each tumor was obtained compared with its paired normal tissue. A p-value $<0.05$ was considered statistically significant.

The levels of phospho-JNK/SAPK ${ }^{\mathrm{p} 46 / \mathrm{p} 54}$ were also assayed using the PathScan Phospho-SAPK/JNK (specific for Thr183 and Tyr185 on JNK 1,2) sandwich ELISA kit (cell signaling). Procedures employed for this assay imply that a mouse monoclonal antibody is coated onto the microwells. After incubation with cell lysates, both nonphospho- and phosphoJNK/SAPK proteins are captured by the coated antibody. Following extensive washing, a phospho-JNK/SAPK antibody is added to detect the captured phospho-JNK/SAPK protein and an HRP-linked anti-rabbit antibody is then used to recognize the bound detection.

Immunohistochemical analysis for activated MAPK was performed on formalin-fixed, paraffin-embedded sections of tumor and paired control samples. Endogenous peroxidase activity was quenched using a 5-min incubation step with $3 \%$ $\mathrm{H}_{2} \mathrm{O}_{2}$ in PBS. Non-specific binding sites were blocked by incubation with $5 \%$ BSA. Slides were incubated at $4{ }^{\circ} \mathrm{C}$ overnight with the primary antibody that specifically recognizes each phospho-MAPK. The primary antibody was detected using biotinylated secondary antibodies and this was followed by the addition of streptavidin-horseradish peroxidase conjugate complex. The complex was visualized by the enzymatic reduction of 3'3 diaminobenzidine tetrahydrochrolide (DAB) substrate.

\section{Results}

Immunoblot analysis of MAPKs. In this study, twenty-seven SCC of the larynx were compared to paired perilesional normal laryngeal tissue for phosphorylation status of several mitogenic signaling effectors. We evaluated both the total levels of p38, JNK/SAPK ${ }^{\mathrm{p} 46 / \mathrm{p} 54}$, ERK1/2 ${ }^{\mathrm{p} 44 / \mathrm{p} 42}$ and the levels of the active (phosphorylated) form of the same proteins by 

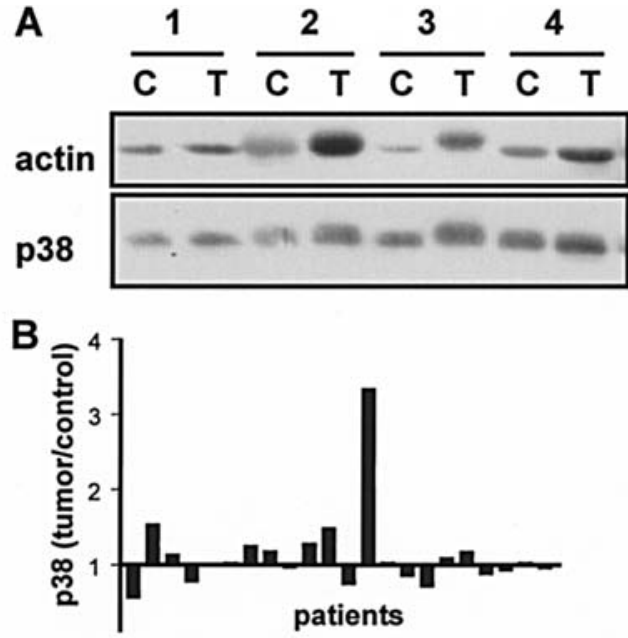

Figure 1. Expression of the total amount of p38 in laryngeal tumors. Comparing tumors $(\mathrm{T})$ with their paired normal tissues $(\mathrm{C})$ no statistically significant difference emerges. (A) Representative immunoblot analysis showing expression of the total amount of p38 in laryngeal tumors and paired normal tissues. (B) Expression of total amount of p38 was quantified by densitometric analysis of autoluminographs. To control for differences in protein loading, we normalized the expression of the total amount of $\mathrm{p} 38$ to expression of actin in each sample. We then set the levels of expression of total amount of p38 in each normal tissue to 1 , and thus obtained the relative change in expression of the total amount of p38 in each tumor compared with its paired normal tissue. The average relative change in expression for each tumor/normal tissue pair is shown.

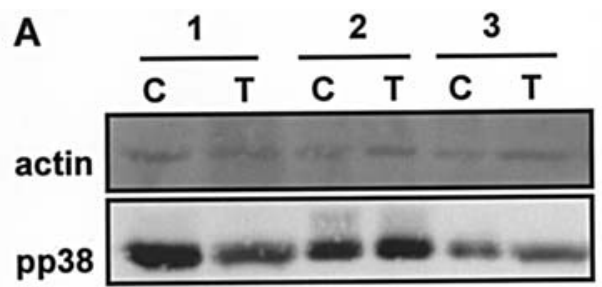

B

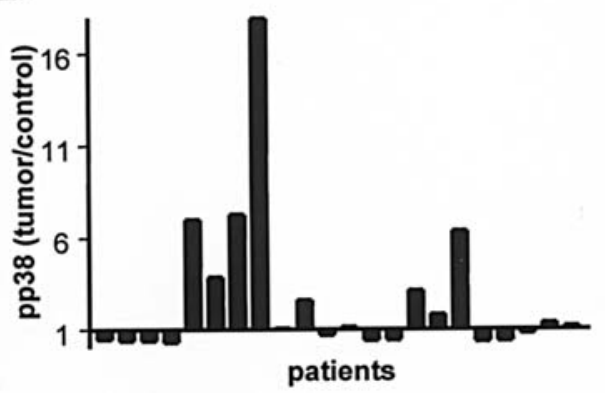

Figure 2. Expression of phospho-p38 in laryngeal tumors. Comparing tumors (T) with their paired normal tissues (C) no statistically significant difference emerges. (A) Representative immunoblot analysis showing expression of phospho-p38 in laryngeal tumors and paired normal tissues. (B) Expression of phospho-p38 was quantified by densitometric analysis of autoluminographs. To control for differences in protein loading, we normalized the expression of phospho-p38 to the expression of actin in each sample. We then set the levels of expression of phospho-p38 in each normal tissue to 1 , and thus obtained the relative change in expression of phospho-p38 in each tumor compared with its paired normal tissue. The average relative change in expression for each tumor/normal tissue pair is shown.

immunoblot analysis. The different MAPK isoforms were detected in every tissue sample examined. In some samples, immunoblotting did not result in satisfactory products to be

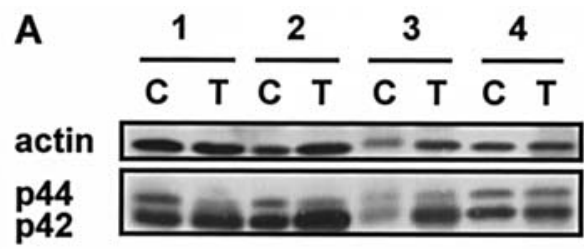

B

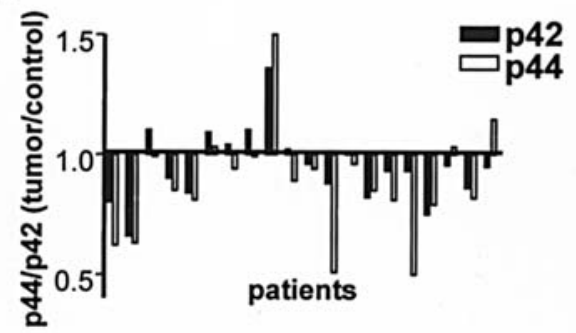

Figure 3. Expression of the total amount of ERK1/2 $44 / \mathrm{p} 42$ in laryngeal tumors. Comparing tumors (T) with their paired normal tissues (C) no statistically significant difference emerges. (A) Representative immunoblot analysis showing expression of the total amount of ERK $1 / 2^{\mathrm{p} 44 / \mathrm{p} 42}$ in laryngeal tumors and paired normal tissues. (B) Expression of the total amount of ERK $1 / 2^{\mathrm{p} 44 / \mathrm{p} 42}$ was quantified by densitometric analysis of autoluminographs. To control for differences in protein loading, we normalized the expression of the total amount of ERK $1 / 2^{\mathrm{p} 44 / \mathrm{p} 42}$ to expression of actin in each sample. We then set the levels of expression of total amount of ERK $1 / 2^{\mathrm{p} 44 / \mathrm{p} 42}$ in each normal tissue to 1 , and thus obtained the relative change in expression of the total amount of ERK1/2 $2^{\mathrm{p} 4 / \mathrm{p} 42}$ in each tumor compared with its paired normal tissue. The average relative change in expression for each tumor/normal tissue pair is shown.

efficiently quantified by densitometric analysis and these were not considered. For this reason, the number of cases analyzed for the different signaling effectors did not coincide. For both total and active (phosphorylated) p38 proteins, values related to the tumor samples were scattered compared with those of normal tissues (Fig. 1B and Fig. 2B). However, no statistically significant differences in the total amount (Fig. 1A) and in the phosphorylation (Fig. 2A) of p38 were evident between tumors and normal samples. Immunoblotting did not show statistically significant difference for the total amount of either isoform of JNK/SAPK (p46 and p54) (data not shown) while the analysis of the activated forms did not permit us to obtain reliable results. Consequently, the activation of both JNK/SAPK isoforms was analyzed by an ELISA assay. The results obtained did not show statistically significant differences between tumor and normal laryngeal samples. The level of expression of phospho-JNK/SAPK was $0.437 \pm 0.357$ (mean OD $\pm \mathrm{SD}$ ) in the controls and $0.509 \pm 0.3164$ (mean OD \pm SD) in the tumors, $p>0.05$.

On the contrary, a statistically significant difference was observed analyzing the amount of phospho-ERK $1 / 2$ pattern in tumors compared with normal tissues. The levels of total protein for both ERK1(p44) and ERK2(p42) were not statistically different between tumor and normal samples (Fig. 3A and B). Analysis of the levels of activated (phosphorylated) forms of ERK1/2 showed a statistically significant reduction for both isoforms in tumor samples compared to the normal mucosa (Fig. 4A and B). The average phospho-ERK1 expression level in cancerous and in normal tissues was $0.8081 \pm 0.41$ (mean $\mathrm{RE} \pm \mathrm{SD}$ ) and $1.354 \pm 0.369$ (mean $\mathrm{RE} \pm \mathrm{SD}$ ) respectively, $\mathrm{p}<0.05$. The 

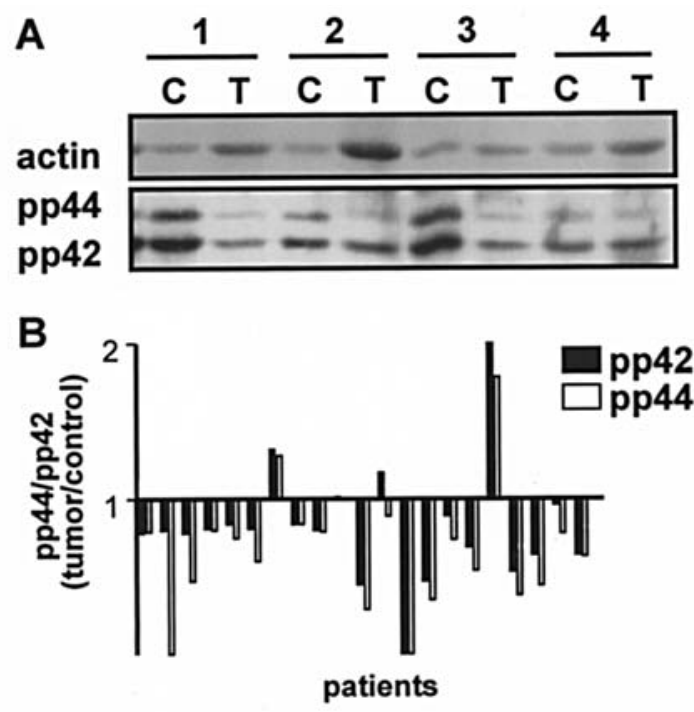

Figure 4. Expression of phospho-ERK1/2 $2^{\mathrm{p} 4 / \mathrm{p} 42}$ is statistically reduced in laryngeal tumors (T) compared to their paired normal tissues (C). (A) Representative immunoblot analysis showing expression of phosphoERK $1 / 2^{\mathrm{p} 4 / \mathrm{p} 42}$ in laryngeal tumors and paired normal tissues. (B) Expression of phospho-ERK $1 / 2^{\mathrm{p} 4 / \mathrm{p} 42}$ was quantified by densitometric analysis of autoluminographs. To control for differences in protein loading, we normalized the expression of phospho-ERK $1 / 2^{\mathrm{p} 44 / \mathrm{p} 42}$ to the expression of actin in each sample. We then set the levels of expression of phosphoERK $1 / 2^{\text {p44/p42 }}$ in each normal tissue to 1 , and thus obtained the relative change in expression of phospho-ERK $1 / 2^{\mathrm{p} 44 / p^{42}}$ in each tumor compared with its paired normal tissue. The average relative change in expression for each tumor/normal tissue pair is shown.

average phospho-ERK2 expression resulted in $1.569 \pm 0.482$ (mean $\mathrm{RE} \pm \mathrm{SD}$ ) in the control tissues and 1.177 \pm 0.486 in the tumors, $\mathrm{p}<0.05$. As shown in Fig. 4B, modulation of the two isoforms, ERK1 and ERK2, in the tumor samples were in strict correlation. In almost all the tumors the phosphorylation of both isoforms was reduced compared to the control samples and the rate of reduction was similar for both isoforms. Only 2 patients showed an increase in phosphorylation of both isoforms in the tumor samples compared to the corresponding control tissue. One patient showed an opposite modulation of the two different isoforms.

We also evaluated whether there might be a correlation between tumor grading and specific Western blot patterns, but no significant findings were found (data not shown).

Immunohistochemical analysis of activated MAPKs. In order to evaluate the cellular localization of the activated MAPKs, we performed an immunohistochemical analysis for the phosphorylated forms of MAPKs. This analysis showed that all the activated MAPKs (p38, JNK/SAPK ${ }^{\mathrm{p} 46 / \mathrm{p} 54}$ and ERK1/2 ${ }^{\mathrm{p} 4 / \mathrm{p} 42}$ ) were expressed in both laryngeal tumors (Fig. 5) and normal tissue (data not shown). No obvious difference of cellular localization for all the activated MAPKs was demonstrated between the tumor and normal tissues. Activated ERK1/2 $2^{\text {p4/p42 }}$ and activated p38 showed a nucleo-cytoplasmatic distribution whereas JNK/SAPK ${ }^{\mathrm{p} 4 / \mathrm{p} 54}$ was localized into the cytoplasmatic membrane. This distribution was common to normal and tumor tissues.
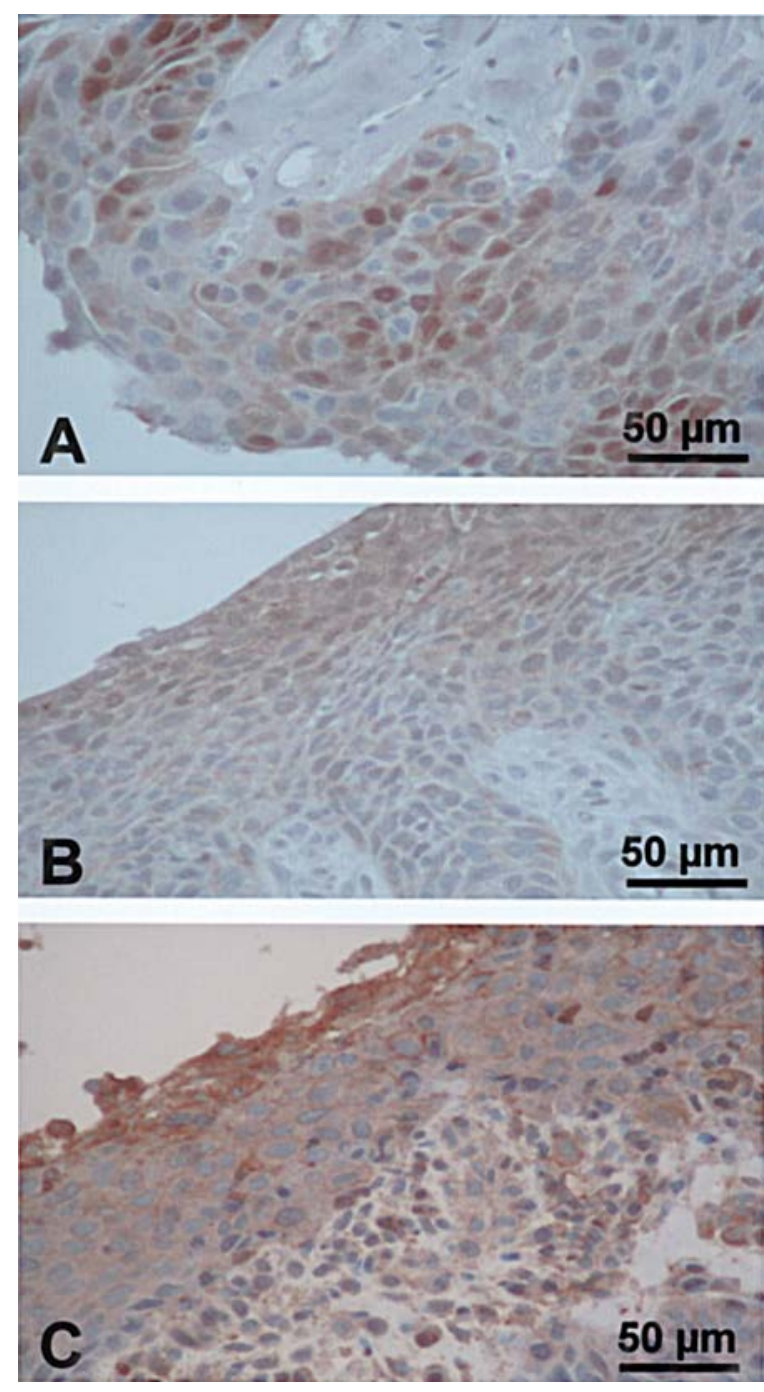

Figure 5. Cellular localization of activated (phosphorylated) forms of MAPK in laryngeal tumors. Activated ERK1/2 $44 / \mathrm{p} 42(\mathrm{~A})$ and activated p38 (B) shows a nucleo-cytoplasmatic distribution whereas activated $\mathrm{JNK} / \mathrm{SAPK}^{\mathrm{p} 46 / \mathrm{p} 54}(\mathrm{C})$ are localized into the cytoplasmatic membrane.

\section{Discussion}

This study analyzed the modulation of MAPKs in SCC of the larynx and demonstrated that ERK $1 / 22^{\mathrm{p} 44 / \mathrm{p} 42}$ degree of phosphorylation may be a possible marker of malignancy. Indeed, in the tumor specimens evaluated, the total amount of MAPK proteins was not significantly different from corresponding control tissues. Conversely, a significant reduction of the phosphorylated form of both ERK1 and ERK2 was observed in the malignant samples.

The present data are in complete agreement with the findings from Tosi et al (8) that, in the heterogeneous group of HNSCC, found a significant reduction of the phosphorylated forms of two different families of key signalling pathway downstream of the activated receptors, MAPK and Akt, whereas PKCs showed no differences from the control samples. Conversely, our results are in disagreement with previously published data from Albanell et al (9) who found that activated forms of ERK1 and ERK2 localized predominantly in the nucleus of tumor cells in a series of HNSCC. There are 
several potential explanations for these controversies: i) the tumor series analyzed by Albanell et al (9) included oral cavity and pharyngeal carcinomas and no laryngeal cancers while this type of tumor was predominant in the series of patients reported by Tosi et al (8) and is the unique type analyzed in the present study, ii) although the antibody used was presumably the same in all three studies, the techniques employed to evaluate the phosphorylated status were different, being mostly based on immunostaining in the case of Albanell et al (9) and by immunoblotting in the other two (8), iii), the study by Albanell et al (9) was probably limited by the lack of comparison between the pathological samples and the corresponding normal tissue that, according to the present study, is characterized by a consistent amount of phosphorylated MAPK. Whether discrepancies in results obtained are to be attributed to site-specific differences of the tumors evaluated or to other factors, for example methodological procedures, is still to be established.

Changes in the activation status of signal pathway circuits that regulate downstream cell cycle progression ultimately result in the loss of cell growth control and the net accumulation of neoplastic cells (11). In this context, the role of the MAPK cascades in controlling cell proliferation may be tissue- or cell type-, as well as stage-specific, and in some cases, may involve yet unidentified MAPK pathways (16). Increased expression of ERK is observed in several kinds of human cancers compared with paired normal tissues $(21,22)$. Conversely, similarly to that observed in the present study for SCC of the larynx, decreased levels of phosphorylated forms of MAPKs have been associated with prostate cancer. Moreover, the high level of phosphorylated ERK present in the normal epithelium, was followed by a gradual decline in disease progression (23). This cancer-specific opposite behaviour underlies an essential difference in the regulation of growth. In line with this observation, controversial findings have been reported in relation to the regulation and function of ERKs in mammalian mitosis (24). While the biochemical activities of endogenous ERK1 and ERK2 have been shown to decrease as cells enter mitosis (25), other studies have concluded that the activation of the ERK cascade is required for normal progression to mitosis (26). Moreover, it should be noted that in important experimental systems such as neuronal cells, the activation of ERK leads to terminal differentiation (27). Thus, one may speculate that the decreased activity of ERKs in some forms of cancer could be the cause of cellular dedifferentiation. Finally, the different behaviour displayed by different tumors is thought to be dependent on the integration of multiple signals from the numerous transduction pathways. The activation of different pathways can generate opposing signals regulating survival, proliferation and programmed cell death. In prostate cancer (23) a significant surge in phosphorylated Akt, providing an important cell survival signal, was found concomitant with a decreased phoshorylation of ERK. In this context, it has been recently underlined that specific signalling pathways (for instance, the Ras-Rac cascade) can be regulated at distinct and differentially regulated steps of the cascade with the consequence of a different duration of the signalling within a cell (28). The activation of Ras leads to stimulation of ERK kinases as an early event consequent to the receptor stimulation while, in the late event, the termination of the Ras activation leads to the switch-off of the upstream signalling and the activation of other pathways.

In conclusion, the results of the present study support previous published findings indicating significant alterations in the MAPK activation in SCC of the larynx. A significant decrease in ERK1 and ERK2 phosphorylation was found. The functional significance of these results and the interplay among different signalling pathways in these tumors warrant further investigations aimed at correlating the decrease of phospho-ERKs with the metalloproteinase MMP-2 activity which, as recently suggested by Munshi et al (29), is induced by TGF- 31 through the negative mediation of ERK1/2.

\section{Acknowledgements}

This study was supported by a research grant from MIUR/ COFIN 2003069877. D.M is supported by a grant from FERB.

\section{References}

1. Parkin DM, Bray F, Ferlay J and Pisani P: Global cancer statistics, 2002. CA Cancer J Clin 55: 74-108, 2005.

2. IARC, Working group on the evaluation of carcinogenic risks to humans: Tobacco smoking. In: IARC Monographs on the evaluation of carcinogenic risks to humans. IARC 38, Lyon, 1988 .

3. IARC, Working group on the evaluation of carcinogenic risks to humans: Alcohol drinking. In: IARC Monographs on the evaluation of carcinogenic risks to humans. IARC 44, Lyon, 1998.

4. Paulovich AG, Toczyski DP and Hartwell LH: When checkpoints fail. Cell 88: 315-321, 1997.

5. Evan GI and Vousden KH: Proliferation, cell cycle and apoptosis in cancer. Nature 411: 342-328, 2001.

6. Brachman DG: Molecular biology of head and neck cancer. Semin Oncol 21: 320-329, 1994.

7. Das BR and Nagpal JK: Understanding the biology of oral cancer. Med Sci Monit 8: 258-267, 2002.

8. Tosi L, Rinaldi E, Carinci F, Farina A, Pastore A, Pelucchi S, Cassano L, Evangelisti R, Carinci P and Volinia S: Akt, protein kinase $\mathrm{C}$, and mitogen-activated protein kinase phosphorylation status in head and neck squamous cell carcinoma. Head Neck 27: 130-137, 2005.

9. Albanell J, Codony-Servat J, Rojo F, Del Campo JM, Sauleda S, Anido J, Raspall G, Giralt J, Rosello J, Nicholson RI, Mendelsohn J and Baselga J: Activated extracellular signalregulated kinases: association with epidermal growth factor receptor/transforming growth factor alpha expression in head and neck squamous carcinoma and inhibition by anti-epidermal growth factor receptor treatments. Cancer Res 61: 6500-6510, 2001 .

10. Greenberg AK, Basu S, Hu J, Yie TA, Tchou-Wong KM, Rom WN and Lee TC: Selective p38 activation in human non-small cell lung cancer. Am J Respir Cell Mol Biol 26: 558-564, 2002.

11. Freeman SM and Whartenby KA: The role of the mitogenactivated protein kinase cellular signaling pathway in tumor cell survival and apoptosis. Drug News Perspect 17: 237-242, 2004.

12. Santen RJ, Song RX, McPherson R, Kumar R, Adam L, Jeng MH and Yue W: The role of mitogen-activated protein (MAP) kinase in breast cancer. J Steroid Biochem Mol Biol 80: 239-256, 2002.

13. Zhang $\mathrm{W}$ and Liu HT: MAPK signal pathways in the regulation of cell proliferation in mammalian cells. Cell Res 12: 9-18, 2002.

14. Chen Z, Gibson TB, Robinson F, Silvestro L, Pearson G, Xu B, Wright A, Vanderbilt $\mathrm{C}$ and Cobb $\mathrm{MH}$ : MAP kinases. Chem Rev 101: 2449-2476, 2001.

15. Lee SH, Lee JW, Soung YH, Kim SY, Nam SW, Park WS, Kim SH, Yoo NJ and Lee JY: Colorectal tumors frequently express phosphorylated mitogen-activated protein kinase. APMIS 112: 233-238, 2004. 
16. Engelberg D: Stress-activated protein kinases - tumor suppressors or tumor initiators? Semin Cancer Biol 14: 271-282, 2004.

17. Yang YM, Bost F, Charbono W, Dean N, McKay R, Rhim JS, Depatie C and Mercola D: C-Jun NH(2)-terminal kinase mediates proliferation and tumor growth of human prostate carcinoma. Clin Cancer Res 9: 391-401, 2003.

18. Villa D, Miloso M, Nicolini G, Rigolio R, Villa A, Cavaletti G and Tredici G: Low-dose cisplatin protects human neuroblastoma SH-SY5Y cells from paclitaxel-induced apoptosis. Mol Cancer Ther 4: 1439-1447, 2005.

19. Nicolini G, Rigolio R, Scuteri A, Miloso M, Saccomanno D, Cavaletti G and Tredici G: Effect of trans-resveratrol on signal transduction pathways involved in paclitaxel-induced apoptosis in human neuroblastoma SH-SY5Y cells. Neurochem Int 42: 419-429, 2003.

20. Zarubin $\mathrm{T}$ and Han $\mathrm{J}$ : Activation and signaling of the p38 MAP kinase pathway. Cell Res 15: 11-18, 2005.

21. Sivaraman VS, Wang H, Nuovo GJ and Malbon CC: Hyperexpression of mitogen-activated protein kinase in human breast cancer. J Clin Invest 99: 1478-1483,1997.

22. Mishima K, Yamada E, Masui K, Shimokawara T, Takayama K, Sugimura M and Ichijima K: Overexpression of the ERK/MAP kinases in oral squamous cell carcinoma. Mod Pathol 11: 886-891, 1998.

23. Paweletz CP, Charbonea L, Bichsel VE, Simone NL, Chen T, Gillespie JW, Emmert-Buck MR, Roth MJ, Petricoin III EF and Liotta LA: Reverse phase protein microarrays which capture disease progression show activation of pro-survival pathways at the cancer invasion front. Oncogene 20: 1981-1989, 2001.
24. Minshull J, Sun H, Tonks NK and Murray AW: A MAP kinasedependent spindle assembly checkpoint in Xenopus egg extracts. Cell 79: 475-486, 1994.

25. Edelmann HM, Kuhne C, Petritsch C and Ballou LM: Cell cycle regulation of p70 S6 kinase and p42/p44 mitogen-activated protein kinases in Swiss mouse 3T3 fibroblasts. J Biol Chem 271: 963-971, 1996.

26. Hayne C, Tzivion G and Luo Z: Raf-1/MEK/MAPK pathway is necessary for the $\mathrm{G} 2 / \mathrm{M}$ transition induced by nocodazole. J Biol Chem 275: 31876-31882, 2000.

27. Cowley S, Paterson H, Kemp P and Marshall CJ: Activation of MAP kinase kinase is necessary and sufficient for PC12 differentiation and for transformation of NIH 3T3 cells. Cell 77: 841-852, 1994.

28. Innocenti M, Tenca P, Frittoli E, Faretta M, Tocchetti A, Di Fiore PP and Scita G: Mechanisms through which Sos-1 coordinates the activation of Ras and Rac. J Cell Biol 156: $125-136,2002$.

29. Munshi HG, Wu YI, Mukhopadhyay S, Ottaviano AJ, Sassano A, Koblinski JE, Platanias LC and Stack MS: Differential regulation of membrane type 1 -matrix metalloproteinase activity by ERK 1/2 and p38 MAPK-modulated tissue inhibitor of metalloproteinases 2 expression controls transforming growth factor-ß1-induced pericellular collagenolysis. J Biol Chem 279: 39042-39050, 2004. 